\title{
Assessing the Flowering Genetic Regulatory Network in Neotropical Orchids ${ }^{+}$
}

\author{
Yesenia Madrigal ${ }^{1}$, Diego Ospina-Zapata ${ }^{1}$, Andrea Ramírez-Ramírez ${ }^{1}$, Juan Fernando Alzate ${ }^{2}$ \\ and Natalia Pabón-Mora ${ }^{1, *}$ \\ 1 Facultad de Ciencias Exactas y Naturales, Instituto de Biología, Universidad de Antioquia, Medellín, \\ Colombia; yesenia.madrigal@udea.edu.co (Y.M.); diego.ospinaz@udea.edu.co (D.O.-Z.); \\ jessicaa.ramirez@udea.edu.co (A.R.-R.) \\ 2 Centro Nacional de Secuenciación Genómica, Sede de Investigación Universitaria, Facultad de Medicina, \\ Universidad de Antioquia, Medellín, Colombia; jfernando.alzate@udea.edu.co \\ * Correspondence: lucia.pabon@udea.edu.co; Tel.: +57-321-772-0164 \\ + Presented at the 1st International Electronic Conference on Plant Science, 1-15 December 2020; Available \\ online: https://iecps2020.sciforum.net/.
}

Published: 30 November 2020

\begin{abstract}
During the reproductive transition in flowering plants a vegetative apical meristem (SAM) transforms into an inflorescence meristem (IM) that forms bracts and flowers. In grasses, like rice, a Genetic Regulatory Network (GRN) controlling reproductive transitions has been identified. It includes the integration of promoters and repressors from different gene lineages with active duplication events during angiosperm diversification. With the objective to understand the evolution and expression of flowering GRN in Orchidaceae, we performed comprehensive phylogenetic analyses of all genes from the flowering GRN and analyzed by RT-PCR the expression of targeted homologs in key developmental stages. Our ML results indicate that FT/TFL1, FD, FLC/FUL, SOC1 and AGL24/SVP gene lineages have been subject to multiple duplications in monocots as well as in Orchidaceae. Conversely, FLC genes are lost in Orchidaceae, suggesting major changes in the repression of flowering. Our studies also show active expression of many target genes in Elleanthus aurantiacus (Orchidoideae) in the SAM and in IM indicating important functions in the reproductive transition. We describe how the flowering GRN in orchids has significant variations in copy number and expression patterns when compared to the canonical rice flowering GRN.
\end{abstract}

Keywords: AGAMOUS LIKE 24; FLOWERING; FLOWERING LOCUS T; FLOWERING LOCUS C; FLOWERING LOCUS D; gene evolution; genetic regulatory network; orchidaceae; SUPPRESSOR OF OVEREXPRESSION OF CONSTANS 1

\section{Introduction}

The floral transition is one of the most important developmental switches in the plant life cycle resulting in the change from vegetative to reproductive phase. In Arabidopsis, the reproductive transition occurs when the vegetative apical meristem (SAM) forming leaves, becomes an inflorescence meristem (IM) that forms bracts and flowers. This process is regulated by endogenous and environmental factors, which merge into four main pathways: photoperiod (light response), vernalization (cold response), autonomous and hormonal signaling [1,2]. In the model monocot, Oryza sativa, the core flowering genetic regulatory network (GRN) relies on early activation of Heading date $3 a$ (Hd3a, a FLOWERING LOCUS T-FT homolog) in short days (SD) [3,4]. The complex between Hd1 (a CO, CONSTANS homolog) and Hd3a plays a critical role in mediating the photoperiod flowering signal [5]. While in SD Hd1 activates FT expression in rice, in long days (LD) Hd1 is converted into a transcriptional repressor [5]. However on LD rice cultivars, RICE 
FLOWERING LOCUS T1 (RFT1), an Hd3a paralog, is responsible for floral induction [3]. In SD, After the first FT signaling, FLOWERING LOCUS D (FD, a bZIP homolog) transcription factor in rice, OsFD1, interacts with Hd3a via the 14-3-3 proteins to form a florigen activation complex (FAC) [6]. The FAC induces the transcription of OsMADS14 and OsMADS15 (the AP1/FUL homologs) in the shoot apex during floral transition [6-8]. On the other hand, OsMADS50 and OsMADS51 (the SOC1 homologs), OsMADS22, OsMADS47 and OsMADS55 (the AGL24/SVP homologs) control floral meristem identity, but only OsMADS55 represses flowering [9-11]. A relatively similar flowering GRN is in place across grasses [12-14]. However, crown pooids like wheat (Triticum monococcum, Triticum aestivum) and barley (Hordeum vulgare), also have vernalization responsiveness determined by allelic variation at the VERNALIZATION1 (VRN1, an AP1/FUL homolog) and/or VRN2 (a CO-like homolog) loci [15-17]. VRN2 alleles repress flowering by direct or indirect repression of $V R N 1$ alleles under LD [18]. In addition, during vernalization and/or exposure to SD, VRN2 transcription is reduced, resulting in an up-regulation of VRN1 and triggering flowering [15,17].

Although the flowering GRN has been well studied in grasses, little is known about the genetic mechanisms of flowering in non-model monocots, including orchids. The isolation and characterization of some flowering controlling transcription factors have been done in commercial, mostly temperate orchids like Cymbidium, Dendrobium, Oncidium and Phalaenopsis: here, homologs of $F T$ or SOC1 genes play an important role in promoting flowering $[19,20]$. Nevertheless, comprehensive phylogenetic analyses for all gene lineages involved in the flowering GRN are lacking, and as a consequence, few homologs have been studied, sometimes with unclear affiliation to a specific clade. This is particularly problematic considering that whole genome duplication (WGD) events are abundant in monocots. In turn, gene copy number and homology for all copies needs to be established prior to expression and functional characterization of the flowering GRN. Our goal is to evaluate the evolution of the flowering GRN in the Orchidaceae (ca. 25,000 species), one of the most diverse groups of ornamental angiosperms. Here we use reference transcriptomes from 13 neotropical orchid species to find homologs from the transcription factors known to control flowering and perform comprehensive ML phylogenetic analyses to understand the evolution of all gene lineages involved in the reproductive transition. Our ML results indicate that FT/TFL1, FD, FLC/FUL, SOC1 and AGL24/SVP gene lineages have been subject to multiple duplications in monocots, as well as in Orchidaceae. We also show that FLC genes are lost in orchids. Finally we evlaute expression of all target genes in Elleanthus aurantiacus, a tropical and terrestrial member of the Orchidoideae (Orchidaceae) and show active expression of several factors in the SAM and IM indicating important functions in the reproductive transition. We show that the flowering GRN in orchids has significant variations in copy number and expression patterns when compared to the canonical rice flowering GRN.

\section{Experiments}

\subsection{Phylogenetic Analyses of Flowering Candidate Genes}

In order to analyze the evolution of flowering-related gene lineages FD, FLC/FUL and SOC1 and identify putative duplication events, we performed searches for gene homologs of all candidate genes using tBLASTX tools. Searches were done in our own reference transcriptomes as well as in the Orchidstra and OrchidBase which serve as repositories for orchid genomes and transcriptomes [21,22]. The queries were FD, FUL and SOC1 homologs from Arabidopsis, orchids and rice. Dteiled methodology for phylogenetic analyses can be found in [23] and [26].

\subsection{Morpho-Anatomical Characterization of the Flowering Transition in Orchidaceae}

In order to establish changes in size, and the initiation of lateral organs as well as new morphological features occurring during flowering transition in Elleanthus aurantiacus light and scanning electron microscopy were used. Detailed steps for sample processing follow [24]. 


\subsection{RT-PCR Expression Analysis of GRN Candidate Genes}

RT-PCR using cDNA from dissected parts in Elleanthus aurantiacus was performed to evaluate the expression patterns of flowering gene homologs. Dissections follow [23]. For the amplification of each homolog, specific primers were designed for each copy avoiding conserved domains and sometimes including either the $3^{\prime}$ or $5^{\prime}$ UTRs (Table A1). Amplification reactions were done following [25]. ACTIN was used as a positive control.

\section{Results}

\subsection{Flowering GRN Genes Have Undergone Multiple Duplication Events}

The BLAST search resulted in the recovery of FT, FD, FLC/FUL, SOC1 and AGL24/SVP homologs in all orchid repositories, including por own reference transcriptomes from neotropical orchids (Table 1) as well as other publicly available angiosperm databases used. All sequences were evaluated using ML phylogenetic analyses and resulted in a comprehensive assesment of the flowering GRN evolution in Orchidaceae.

Table 1. Neotropical orchid species with available reference transcriptome $s^{1}$ and their number of GRN homologs included in ML phylogenetic analyses.

\begin{tabular}{|c|c|c|c|c|c|c|c|c|c|c|c|}
\hline Species & \multicolumn{2}{|c|}{$F T$} & \multicolumn{2}{|c|}{$F D$} & \multicolumn{3}{|c|}{ FUL } & \multicolumn{2}{|c|}{ SOC1 } & \multicolumn{2}{|c|}{ AGL24/SVP } \\
\hline & $\begin{array}{l}3 \\
\stackrel{3}{\circ} \\
\text { 勇 }\end{array}$ & \begin{tabular}{l}
3 \\
\multirow{2}{3}{} \\
\multirow{2}{*}{} \\
$N$
\end{tabular} & 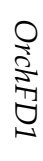 & 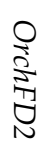 & $\underset{Z}{\grave{Z}}$ & 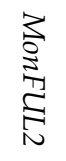 & 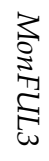 & 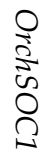 & 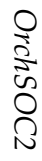 & $\underset{2}{\stackrel{3}{3}}$ & 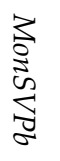 \\
\hline Cattleya trianae & 2 & 1 & 1 & 1 & 0 & 3 & 2 & 2 & 0 & 1 & 2 \\
\hline Elleanthus aurantiacus ${ }^{2}$ & 3 & 2 & 1 & 1 & 0 & 2 & 0 & 2 & 1 & 3 & 4 \\
\hline Epidendrum fimbriatum & 3 & 5 & 1 & 4 & 0 & 4 & 5 & 2 & 0 & 2 & 8 \\
\hline Gomphichis scaposa & 2 & 3 & 0 & 2 & 0 & 2 & 3 & 2 & 0 & 3 & 4 \\
\hline Masdevalia coccinea "Alba" & 3 & 2 & 2 & 1 & 0 & 2 & 2 & 3 & 0 & 2 & 5 \\
\hline Masdevalia wendlandiana & 5 & 3 & 1 & 1 & 0 & 0 & 2 & 2 & 0 & 1 & 6 \\
\hline Maxilaria aurea & 9 & 3 & 1 & 1 & 0 & 2 & 2 & 4 & 6 & 0 & 3 \\
\hline Miltonia roezli & 6 & 0 & 2 & 1 & 0 & 2 & 2 & 2 & 1 & 1 & 4 \\
\hline Oncidium "Gower Ramsey" & 1 & 1 & 1 & 5 & 0 & 0 & 3 & 5 & 0 & 1 & 3 \\
\hline Oncidium "Twinkle" & 2 & 4 & 6 & 1 & 0 & 1 & 4 & 2 & 0 & 2 & 6 \\
\hline Stelis pusilla & 3 & 4 & 2 & 2 & 0 & 1 & 0 & 2 & 0 & 2 & 3 \\
\hline Tolumnia "Cherry red × Ralph yagh" & 2 & 2 & 3 & 0 & 0 & 1 & 4 & 3 & 2 & 1 & 5 \\
\hline Vanilla aphylla & 12 & 3 & 2 & 2 & 0 & 1 & 0 & 7 & 0 & 5 & 3 \\
\hline
\end{tabular}

${ }^{1}$ Contig statistics for reference transcriptomes available in [23], ${ }^{2}$ Species selected for expression analysis in this study.

A total of 349 PEBP homologs were included to assess the evolution of the FT/TFL1 genes in Orchidaceae. The Amborella trichopoda TFL1 (AmtrTFL1) homolog was used as an outgroup. The topology shows a duplication event prior to angiosperm diversification, resulting in the FT and TFL1 clades [23]. TFL1 genes are either lacking or found scarcely in monocots when compared to eudicots [23]. Conversely, more copies of $F T$ are found when compared to TFL1. FT genes show a duplication prior to angiosperm diversification, which generates clades FT1 and FT2. In monocots, the MonFT1 genes form a monophyletic group and have undergone at least two rounds of duplication, resulting in the MonFT1A, MonF1B and MonFT1C clades respectively. On the other hand, the FT2 genes appear to be exclusive to monocots, being absent in the other angiosperm lineages. These genes were duplicated at least twice in monocots resulting in the MonFT2A, MonFT2B, and MonFT2C (Figure 1a) [23]. 


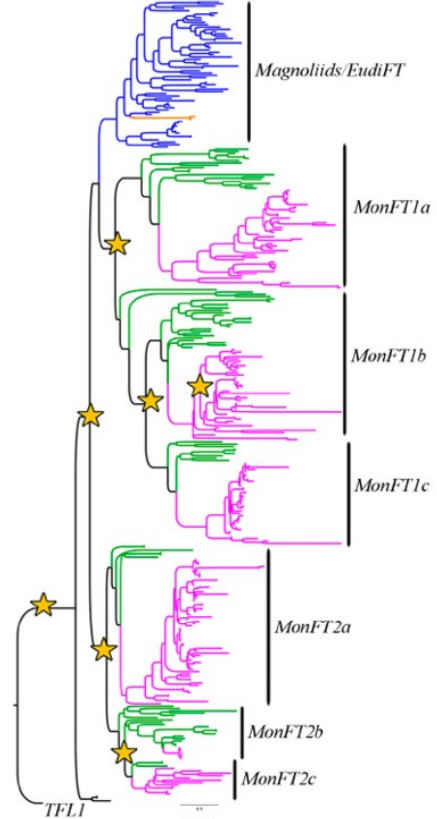

(a)

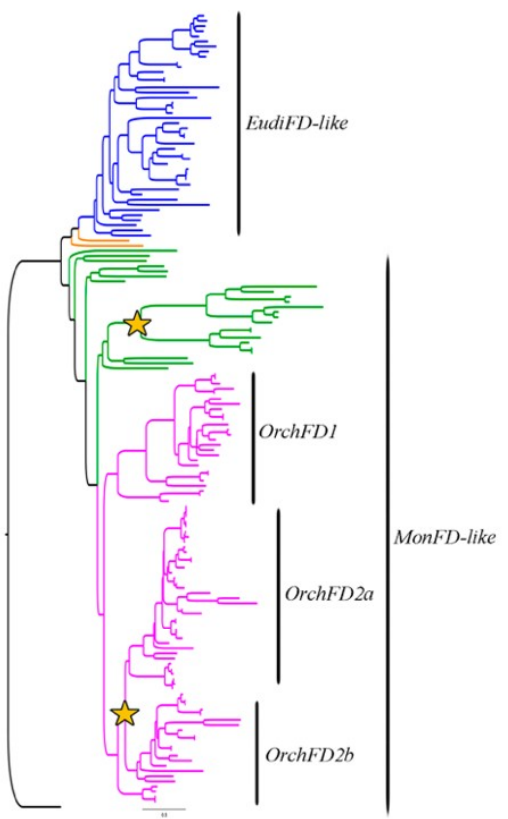

(b)

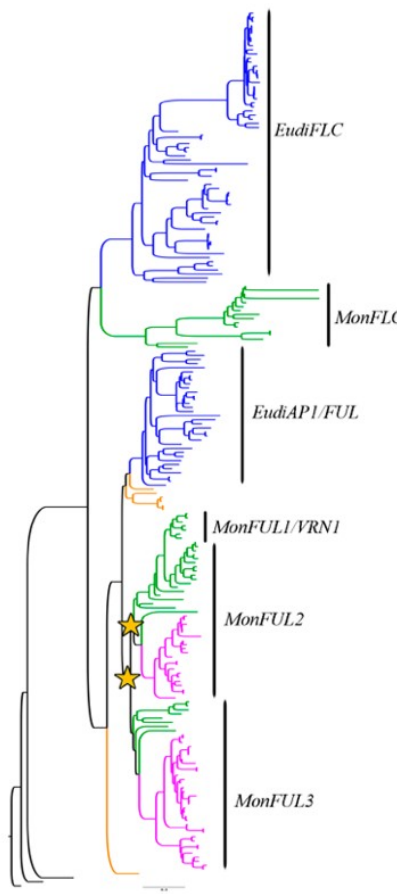

(c)

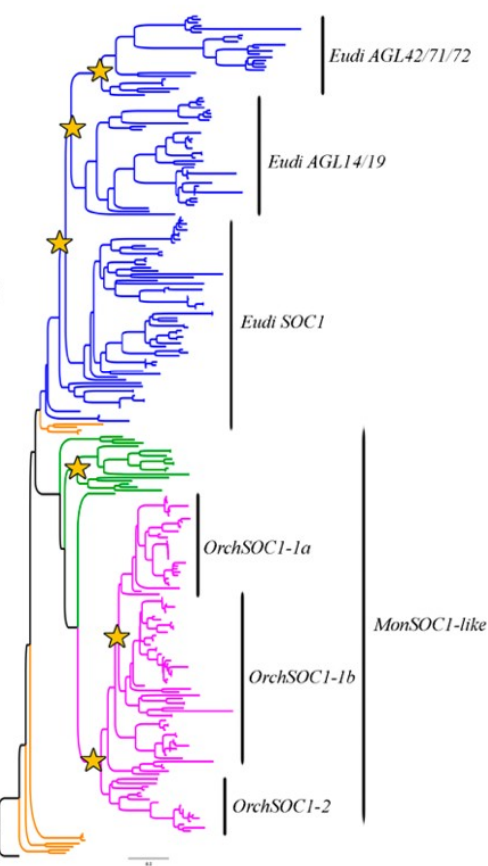

(d)

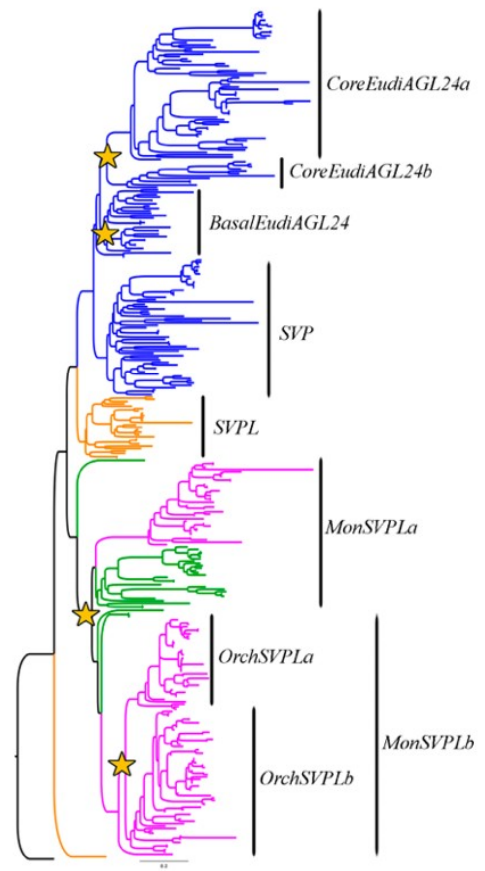

(e)

Eudicots

Non-Orchids Monocots

Orchids

Early divergent angiosperms

Duplication event

Figure 1. ML analyses of the flowering GRN in angiosperms with expanded sampling in Orchidaceae. (a) FT genes (PEBP); (b) FD genes (bZip); (c) FLC/FUL genes (MADS-box); (d) SOC1 genes (MADS-box); (e) AGL24/SVP genes (MADS-box). All trees represent summary topologies with the terminal names removed for better visualization. Tree branch colors follow the conventions on the bottom. Stars point to duplication events. Scale: 0.2. FT and AGL24/SVP trees were modified from [23] and [26].

The FD genes (belonging to bZIP family) were analyzed in a matrix of 156 sequences including diverse angiosperm taxa (Figure 2b). The Amborella trichopoda FD homolog (AmtrFD) was used as an outgroup. These genes have undergone specific duplication in Brassicales and Solanales inside core eudicots. In monocots, these genes have undergone at least three duplication events prior to the 
diversification of the Orchidaceae, forming the OrchFD1, OrchFD2a and OrchFD2b clades. Finally, local duplications have also occurred in Poales.
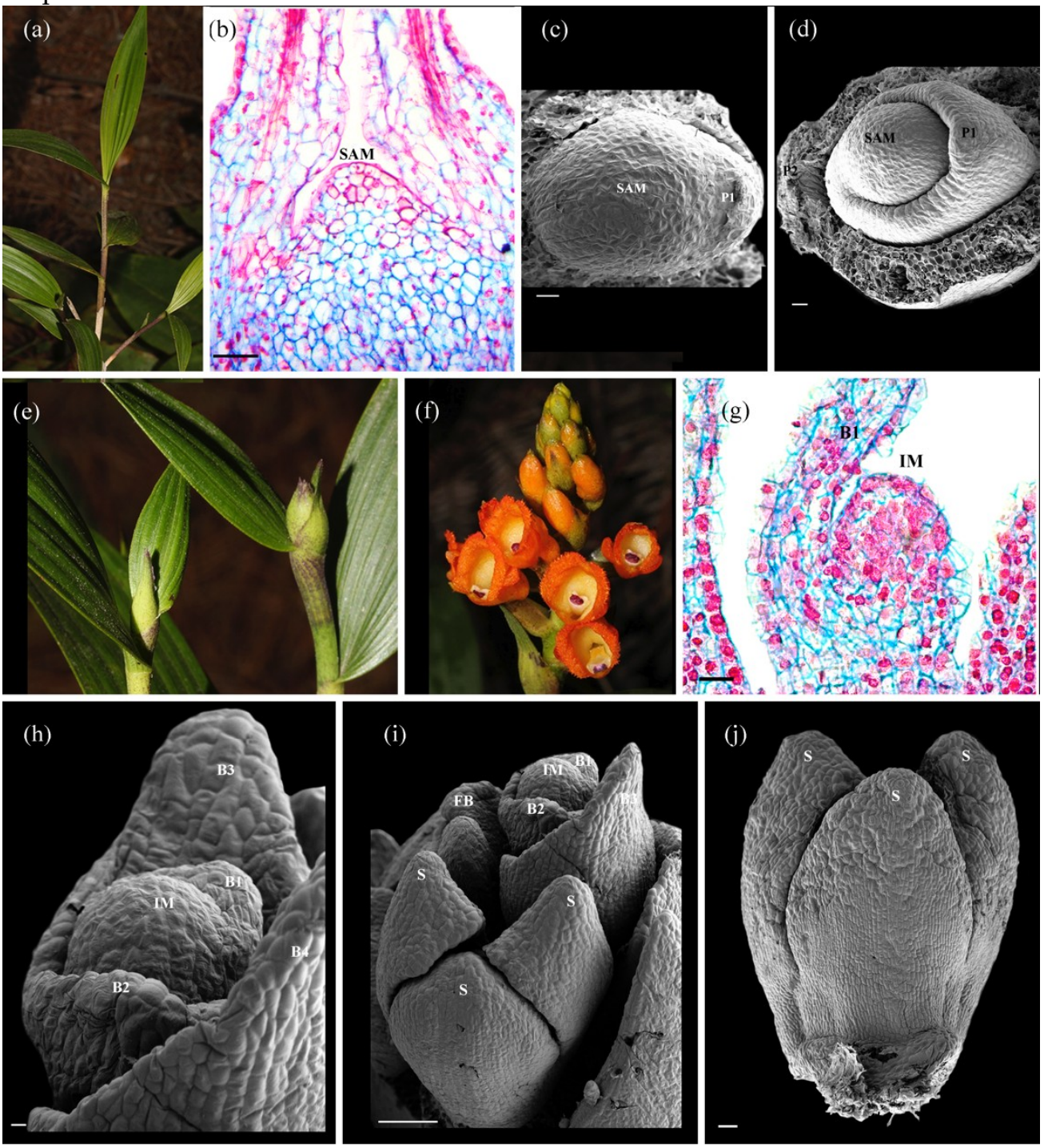

(k)

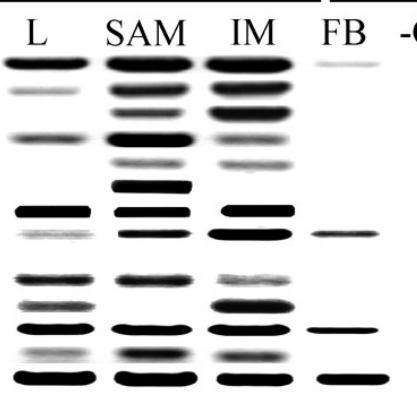

Figure 2. Morpho-anatomical observations and landmarks for developmental stages during flowering transition in Elleanthus aurantiacus, growing at 1700-2400 $\mathrm{m}$ in the Andes, and flowering during the reainy seasons twice a year. (a-d) Plants and apices during vegetative growth; $(\mathbf{e}-\mathbf{j})$ Plants and meristems undergoing reproductive transition. (k) RT-PCR expression patterns of the flowering GRN genes in E. aurantiacus dissected organs, Actin was used as positive control. FT and AGl24/SVP gene expression were taken from [23,26] B: Bract; FB: Floral buttons; IM: Inflorescence meristem; L: leaves; P: Plastochron; S: Sepal; SAM: Vegetative meristem; -c: negative control. Scale d $=50 \mu \mathrm{m}$; $\mathrm{e}-\mathrm{g}=20 \mu \mathrm{m}$; $\mathrm{h}=10 \mu \mathrm{m} ; \mathrm{i}-\mathrm{j}=100 \mu \mathrm{m}$.

ML analyses for FLC/FUL (belonging to MADS-box family) were also performed to understand the evolution and the homology of FLC genes in orchids (Figure 1c). An exhaustive search was done 
accross angiosperms resulting in a matrix with 273 putative homologs. The Amborella trichopoda AGL6 homolog (AmtrAGL6) was used as an outgroup. The resulting phylogenetic tree shows that FLC genes are lacking in orchids, while they are still present in Poales. FLC homologs however have extensively diversified in in eudicots. In addition, FUL genes have undergone at least two duplication events in monocots, resulting in the MonFUL1 (also called VRN1 clade), MonFUL2 and MonFUL3 clades. Interestingly, orchids lack homologs in the VRN1 clade and only have FUL2 and FUL3 homologs.

SOC1 gene evolution (belonging to MADS-box family) was also analyzed. The complete matrix comprised 268 angiosperm sequences (Figure 1d). Amborella trichopoda SOC1 homolog (AmtrSOC1) was used as an outgroup. The ML resulting topology shows at least three duplications prior to the diversification of eudicots resulting in the EudiAGL42/71/72, EudiAGL14/19, and EudiSOC1 clades. In monocots, there are three independent duplications prior to the diversification of the Orchidaceae resulting in then OrchSOC1-1a, OrchSOC1-1b and OrchSOC1-2 clades.

Finally, the AGL24/SVP genes (belonging to MADS-box family) were analyzed using a matrix of 363 sequences (Figure 1e) [26]. The Amborella trichopoda SVP homolog (AmtrSVP) was used as an outgroup. The topology shows a duplication prior to the diversification of eudicots resulting in the the AGL24 and SVP clades. Additional duplications have occurred for AGL24 in eudicots resulting in the CoreeudiAGL24a-b clades. Early diverging angiosperms and monocots only have pre-duplication copies. However, at least one independent duplication has occurred in monocots resulting in the MonSVPLa and MonSVPLb clades, and two additional duplications have occured in MonSVPLa, generating the orchid specific OrchSVPLa and OrchSVPLb clades.

\subsection{The Flowering Transition in Orchidaceae Recruits Several Flowering GRN Genes, Actively Expressed in} the SAM and the IM

Morpho-anatomical analyses in Ellenathus aurantiacus (Orchidioideae, Orchidaceae) show that vegetative growth can occur until plants reach ca. $1.5 \mathrm{~m}$ tall (Figure 2a). The IM starts to differentiate during the rainy seasons (Figure 2e-f) blooming two times per year and yielding inflorescences of 4 to $10 \mathrm{~cm}$ long. Light and scanning electron microscopy show that the SAM consists of a ca. $150 \mu \mathrm{m}$ in diameter forming in its flanks alternate enveloping leaves (Figure $2 \mathrm{~b}-\mathrm{d}$ ). During the floral transition, the IM narrows down to ca. $100 \mu \mathrm{m}$ in diameter, and shifts to forming bracts in its flanks with axillary floral meristems (FM) (Figure 2g-j). Each racemose inflorescence forms up to 22-24 flowers.

Expression analyses were performed in dissected organs to understand the possible contribution of the flowering GRN homologs in E. aurantiacus. RT-PCR analyses show a homogeneous expression of the $S O C 1$ genes in vegetative and inflorescence meristems, and greater expression of FD in SAM (Figure 2k). It is noteworthy that copies of $S O C 1$ are also expressed in leaves. None of these genes is expressed in fully diffenretiated floral buds. Additionally, FT1 genes are expressed in the IM, while FT2 genes have wide expression patterns in all tissues analyzed [23]. Finally, from the 7 AGL24/SVP copies, only two are expressed, specifically, MonSVPLa is active in the SAM and OrchSVPLa is expressed in leaves, SAM and IM [26].

\section{Discussion}

Most expression and functional analyses of selected flowering genes have been done in model orchids like Cymbidium, Dendrobium and Phalaenopsis $[19,20,27]$. However, little is know about the evolution of each gene lineage across angiosperms in general and Orchidaceae in particular, as well as about their contribution to flowering in neotropical orchids. Our exhaustive phylogenetic analyses of all flowering genes taking advantage of private and public databases (Figure 1) highlight that the FT, FD, FUL, SOC1 and AGL24/SVP gene lineages have been subject to multiple duplication events in monocots, contrary to what is established in eudicot model species [28-31]. Also, although the Orchidaceae share some duplications with other monocots [32-35], there are additional family exclusive duplications and in turn, orchids have a greater number of gene copies than grasses. It is possible that the increase in copy number is linked to changes in protein structure and, as a consequence, to functional diversification across homologs [23]. One of the major differences we were able to find is the absence of canonical flowering repressors. Contrary to the other lineages, FLC genes 
have only been found in eudicots and Poales [36-39] and are lost in orchids (Figure 1c). The lack of $F L C$ indicates a profiund shift in the vernalization pathway for all orchids, temperate and tropical. It is possible that other genes are being recruited to fill that repressive function when needed.

The observations in E. aurantiacus allow us to conclude that: 1 . Rainy seasons control flowering for this terrestrial orchid species in native environments; 2. The transition from the SAM to the IM triggers the reduction in size of the meristems concomitant with a shift in gene expression; 3 . There is overlapping expression in the SAM and in the IM for the following copies: ElauSOC1-1-3, ElauFD12, ElauSVP2, ElauFT1A, ElauFT1C2, ElauFT2A2 and ElauMFT. Our results suggest important functions for these transcription factors in the reproductive transition in orchids. Endogenous funtional analysis have only been standardized in Dendrobium, where overexpression of DOFT (one of many FT homologs) [40] and DOSOC1 (one of three SOC1 homologs) [41] exhibits earlier flowering than wild-type orchids. These results suggest that both FT and SOC1 genes play an important role in promoting flowering in the Orchidaceae. However, the increase in the gene copy number and our fundings about their expression in SAM and IM imply that functional studies from GRN are necessary to find the floral integrator genes with determining functions in flowering transition in Orchidaceae.

Based on our data, we propose two important assessments about the flowering GRN in Orchidaceae: (1) the genes of interest in orchids have undergone different evolution pathways in comparison with grass model species, due to independent duplication events in each group; (2) the increase number of homologs in orchids makes it difficult to assign a promoter or repressor function and for that directed RNA-seq as well as functional analyses are clue to understand in the flowering mechanisms employed by the Orchidaceae.

\section{Conclusions}

Due to several independent WGD that have occurred inside both Orchidaceae and grasses, the flowering GRN has remarkable changes in the increase of gene copy number in orchids with unknown functions. Functional and comparative analyses are necessary to understand the role of the different homologs in flowering. It is probably that some of the GRN genes would be conserved in orchids, but the other ones probably have changes in function related with flowering repression.

Author Contributions: Y.M. and N.P.-M. conceived and designed the experiments; Y.M., D.O.-Z. and J.A.R.-R. performed the M.L. analysis; J.F. A assembled the reference transcriptomes. All authors analyzed the data, wrote and approved the final manuscript. All authors have read and agreed to the published version of the manuscript.

Acknowledgments: We thank Markus Günther from the Technische Universität Dresden for technical assistance at the SEM facilities. This research was funded by Estrategia de Sostenibilidad 2018-2019 from Universidad de Antioquia, the Convocatoria COLCIENCIAS 808-2018 (código 110180863819 CT 192-2019), the Convocatoria Programáticas 2017-16302 and the 2019 BSA Graduate Student Research Award from the Botanical Society of America.

Conflicts of Interest: The authors declare no conflict of interest. The founding sponsors had no role in the design of the study; in the collection, analyses, or interpretation of data; in the writing of the manuscript, and in the decision to publish the results.

\section{Abbreviations}

The following abbreviations are used in this manuscript:

$\begin{array}{ll}\text { AGL24/SVP } & \text { AGAMOUS LIKE 24/SHORT VEGETATIVE PHASE } \\ \text { FD } & \text { FLOWERING LOCUS D } \\ \text { FLC } & \text { FLOWERING LOCUS C } \\ \text { FT } & \text { Flowering Locus T } \\ \text { FUL } & \text { FRUITFULL } \\ \text { IM } & \text { Inflorescence Meristem } \\ \text { GRN } & \text { Genetic Regulatory Network } \\ \text { ML } & \text { Maximum Likelihood }\end{array}$


SAM Shoot Apical Meristem

SOC1 SUPPRESSOR OF OVEREXPRESSION OF CONSTANS 1

WGD Whole Genome Duplication

\section{Appendix A}

Table A1. Primers used for gene expression analyses. Fwd indicate forward primer. Rev indicate reverse primer.

\begin{tabular}{|c|c|c|}
\hline Primer Name & Sequence & Amplicon Size (bp) \\
\hline ACTIN7a_fwd & GCATTGTGCTTGATTCCGGTGATGGTGT & \multirow{2}{*}{450} \\
\hline ACTIN7a_rev & CCACCTTAATCTTCATGCTGC & \\
\hline ElauSOC1-3_fwd & GGAAAGACGGAGATGAGAC & \multirow{2}{*}{534} \\
\hline ElauSOC1-3_rev & CTTATGCTGATGATTGTCATC & \\
\hline ElauSOC1-1_fwd & GAAGGACGGAGATGAGACG & \multirow{2}{*}{555} \\
\hline ElauSOC1-1_rev & CAGTTCGGTCTCTACATCCT & \\
\hline ElauSOC1-2_fwd & CGGAGATGAAGCGTATAGAA & \multirow{2}{*}{457} \\
\hline ElauSOC1-2_rev & CATCCTTATAGTGGCTATCA & \\
\hline ElauFD2_Rev & AGCGGATGAGGTTCTTTGAA & \multirow{2}{*}{425} \\
\hline ElauFD2_Fwd & CCACCGTGCTTAGCCTTAGT & \\
\hline ElauFD1_Rev & ATAGTGGTGATCGCCTCCTG & \multirow{2}{*}{357} \\
\hline ElauFD1_Fwd & CCCCAAACACCTAAGCGTAA & \\
\hline
\end{tabular}

\section{References}

1. Levy, Y.Y.; Dean, C. The Transition to Flowering. Plant Cell 1998, 10, 1973-1989.

2. Parcy, F. Flowering: A Time for Integration. Int. J. Dev. Biol. 2005, 49, 585-593. doi:10.1387/ijdb.041930fp.

3. Komiya, R.; Ikegami, A.; Tamaki, S.; Yokoi, S.; Shimamoto, K. Hd3a and RFT1 Are Essential for Flowering in Rice. Development 2008, 135, 767-774. doi:10.1242/dev.008631.

4. Tamaki, S.; Matsuo, S.; Wong, H.L.; Yokoi, S.; Shimamoto, K. Hd3a Protein Is a Mobile Flowering Signal in Rice. Science 2007, 316, 1033-1036. doi:10.1126/science.1141753.

5. Kojima, S.; Takahashi, Y.; Kobayashi, Y.; Monna, L.; Sasaki, T.; Araki, T.; Yano, M. Hd3a, a Rice Ortholog of the Arabidopsis FT Gene, Promotes Transition to Flowering Downstream of Hd1 under Short-Day Conditions. Plant Cell Physiol. 2002, 43, 1096-1105.

6. Taoka, K.; Ohki, I.; Tsuji, H.; Furuita, K.; Hayashi, K.; Yanase, T.; Yamaguchi, M.; Nakashima, C.; Purwestri, Y.A.; Tamaki, S.; et al. 14-3-3 Proteins Act as Intracellular Receptors for Rice Hd3a Florigen. Nature 2011, 476, 332-335. doi:10.1038/nature10272.

7. Tsuji, H.; Nakamura, H.; Taoka, K.; Shimamoto, K. Functional Diversification of FD Transcription Factors in Rice, Components of Florigen Activation Complexes. Plant Cell Physiol. 2013, 54, 385-397. doi:10.1093/pcp/pct005.

8. Taoka, K.; Ohki, I.; Tsuji, H.; Kojima, C.; Shimamoto, K. Structure and Function of Florigen and the Receptor Complex. Trends Plant Sci. 2013, 18, 287-294. doi:10.1016/j.tplants.2013.02.002.

9. Fornara, F.; Gregis, V.; Pelucchi, N.; Colombo, L.; Kater, M. The Rice StMADS11-like Genes OsMADS22 and OsMADS47 Cause Floral Reversions in Arabidopsis without Complementing the Svp and Agl24 Mutants. J. Exp. Bot. 2008, 59, 2181-2190. doi:10.1093/jxb/ern083.

10. Lee, J.H.; Park, S.H.; Ahn, J.H. Functional Conservation and Diversification between Rice OsMADS22/OsMADS55 and Arabidopsis SVP Proteins. Plant Sci. 2012, 185-186, 97-104. doi:10.1016/j.plantsci.2011.09.003.

11. Lee, S.; Kim, J.; Han, J.J.; Han, M.J.; An, G. Functional Analyses of the Flowering Time Gene OsMADS50, the Putative Suppressor of Overexpression of CO 1/Agamous-Like 20 (SOC1/AGL20) Ortholog in Rice. Plant J. 2004, 38, 754-764. doi:10.1111/j.1365-313X.2004.02082.x.

12. Qin, Z.; Wu, J.; Geng, S.; Feng, N.; Chen, F.; Kong, X.; Song, G.; Chen, K.; Li, A.; Mao, L.; et al. Regulation of FT Splicing by an Endogenous Cue in Temperate Grasses. Nat. Commun. 2017, 8, 1-12. doi:10.1038/ncomms14320. 
13. Leiboff, S.; Hake, S. Reconstructing the Transcriptional Ontogeny of Maize and Sorghum Supports an Inverse Hourglass Model of Inflorescence Development. Curr. Biol. 2019, 29, 3410-3419.e3. doi:10.1016/j.cub.2019.08.044.

14. Higgins, J.A.; Bailey, P.C.; Laurie, D.A. Comparative Genomics of Flowering Time Pathways Using Brachypodium Distachyon as a Model for the Temperate Grasses. PLoS ONE 2010, 5, e10065. doi:10.1371/journal.pone.0010065.

15. Trevaskis, B.; Bagnall, D.J.; Ellis, M.H.; Peacock, W.J.; Dennis, E.S. MADS Box Genes Control VernalizationInduced Flowering in Cereals. Proc. Natl. Acad. Sci. USA 2003, 100, 13099-13104. doi:10.1073/pnas.1635053100.

16. Trevaskis, B.; Tadege, M.; Hemming, M.N.; Peacock, W.J.; Dennis, E.S.; Sheldon, C. Short Vegetative Phaselike MADS-BOX Genes Inhibit Floral Meristem Identity in Barley. Plant Physiol. 2007, 143, $225-235$. doi:10.1104/pp.106.090860.

17. Preston, J.C.; Kellogg, E.A. Discrete Developmental Roles for Temperate Cereal Grass Vernalization1/Fruitfull-like Genes in Flowering Competency and the Transition to Flowering. Plant Physiol. 2008, 146, 265-276. doi:10.1104/pp.107.109561.

18. Trevaskis, B.; Hemming, M.N.; Dennis, E.S.; Peacock, W.J. The Molecular Basis of Vernalization-Induced Flowering in Cereals. Trends Plant Sci. 2007, 12, 352-357. doi:10.1016/j.tplants.2007.06.010.

19. Wang, H.; Tong, C.; Jang, S. Current Progress in Orchid Flowering / Flower Development Research. Plant Signal. Behav. 2017, 12. doi:10.1080/15592324.2017.1322245.

20. Wang, S.L.; Viswanath, K.K.; Tong, C.G.; An, H.R.; Jang, S.; Chen, F.C. Floral Induction and Flower Development of Orchids. Front. Plant Sci. 2019, 10, 1-15. doi:10.3389/fpls.2019.01258.

21. McGinnis, S.; Madden, T.L. BLAST: At the Core of a Powerful and Diverse Set of Sequence Analysis Tools. Nucleic Acids Res. 2004, 32. doi:10.1093/nar/gkh435.

22. Altschul, S.F.; Gish, W.; Miller, W.; Myers, E.W.; Lipman, D.J. Basic Local Alignment Search Tool. J. Mol. Biol. 1990, 215, 403-410. doi:10.1016/S0022-2836(05)80360-2.

23. Ospina-Zapata, D.A.; Madrigal, Y.; Alzate, J.F.; Pabón-Mora, N. Evolution and Expression of Reproductive Transition Regulatory Genes FT/TFL1 With Emphasis in Selected Neotropical Orchids. Front. Plant Sci. 2020, 11. doi:10.3389/fpls.2020.00469.

24. González, F.; Pabón-Mora, N. Floral Development and Morphoanatomy in the Holoparasitic Pilostyles Boyacensis (Apodanthaceae, Cucurbitales) Reveal Chimeric Half-Staminate and Half-Carpellate Flowers. Int. J. Plant Sci. 2017, 178, 522-536. doi:10.1086/692505.

25. Madrigal, Y.; Alzate, J.F.; González, F.; Pabón-mora, N. Evolution of RADIALIS and DIVARICATA Gene Lineages in Flowering Plants with an Expanded Sampling in Non-Core Eudicots. Am. J. Bot. 2019, 106, 118. doi:10.1002/ajb2.1243.

26. Ramirez-Ramirez, J.A. Evolución y Expersion de Genes MADS-BOX AGL24 (AGAMOUS LIKE 24) y SVP (SHORT VEGETATIVE PHASE) en Orquídeas Selectas Neotropicales. Undergraduate Thesis, BiologyUniversidad de Antioquia, 2020.

27. Teo, Z.W.N.; Zhou, W.; Shen, L. Dissecting the Function of MADS-Box Transcription Factors in Orchid Reproductive Development. Front. Plant Sci. 2019, 10, 1-17. doi:10.3389/fpls.2019.01474.

28. Wickland, D.P.; Hanzawa, Y. The FLOWERING LOCUS T/TERMINAL FLOWER 1 Gene Family: Functional Evolution and Molecular Mechanisms. Mol. Plant 2015, 8, 983-997. doi:10.1016/j.molp.2015.01.007.

29. Fudge, J.B.; Lee, R.H.; Laurie, R.E.; Mysore, K.S.; Wen, J.; Weller, J.L.; Macknight, R.C. Medicago Truncatula SOC1 Genes Are Up-Regulated by Environmental Cues That Promote Flowering. Front. Plant Sci. 2018, 9, 1-11. doi:10.3389/fpls.2018.00496.

30. Preston, J.C.; Zhong, J.; McKeown, M.; den Bakker, M.; Friedman, J. Comparative Transcriptomics Indicates a Role for SHORT VEGETATIVE PHASE (SVP) Genes in Mimulus Guttatus Vernalization Response. G3 Genes Genomes Genet. 2016, 6, 1239-1249. doi:10.1534/g3.115.026468.

31. Smaczniak, C.; Immink, R.G.H.; Angenent, G.C.; Kaufmann, K. Developmental and Evolutionary Diversity of Plant MADS-Domain Factors: Insights from Recent Studies. Development 2012, 139, 3081-3098. doi:10.1242/dev.074674.

32. Chardon, F.; Damerval, C. Phylogenomic Analysis of the PEBP Gene Family in Cereals. J. Mol. Evol. 2005, 61, 579-590. doi:10.1007/s00239-004-0179-4. 
33. Karlgren, A.; Gyllenstrand, N.; Kallmam, T.; Sundstrom, J.F.; Moore, D.; Lascoux, M.; Lagercrantz, U. Evolution of the PEBP Gene Family in Plants: Functional Diversification in Seed Plant Evolution. Plant Physiol. 2011, 156, 1967-1977. doi:10.1104/pp.111.176206.

34. Preston, J.C.; Kellogg, E.A. Reconstructing the Evolutionary History of Paralogous APETALA1/FRUITFULL- like Genes in Grasses (Poaceae). Genetics 2006, 174, 421-437. doi:10.1534/genetics.106.057125.

35. Alter, P.; Bircheneder, S.; Zhou, L.Z.; Schlüter, U.; Gahrtz, M.; Sonnewald, U.; Dresselhaus, T. Flowering Time-Regulated Genes in Maize Include the Transcription Factor ZmMADS1. Plant Physiol. 2016, 172, 389404. doi:10.1104/pp.16.00285.

36. Ruelens, P.; Maagd, R.A. De; Proost, S.; Geuten, K.; Kaufmann, K. FLOWERING LOCUS C in Monocots and the Tandem Origin of Angiosperm-Specific MADS-Box Genes. Nat. Commun. 2013, 4, 2280. doi:10.1038/ncomms3280.

37. Chen, F.; Zhang, X.; Liu, X.; Zhang, L. Evolutionary Analysis of MIKCc -Type MADS-Box Genes in Gymnosperms and Angiosperms. Front. Plant Sci. 2017, 8, 1-11. doi:10.3389/fpls.2017.00895.

38. Ling, A.C.K.; Rozano, L.; Bakar, U.K.A.; Svp, P. Isolation and Phylogenetic Characterisation of LdSVP , SHORT VEGETATIVE PHASE ( SVP ) Homologous Gene from Lansium Domesticum. J. Trop. Agric. Food Sci. 2018, 46, 75-89.

39. Jiao, F.; Pahwa, K.; Manning, M.; Dochy, N.; Geuten, K. Cold Induced Antisense Transcription of FLOWERING LOCUS C in Distant Grasses. Front. Plant Sci. 2019, 10, 1-9. doi:10.3389/fpls.2019.00072.

40. Ding, L.; Wang, Y.; Yu, H. Overexpression of DOSOC1, an Ortholog of Arabidopsis SOC1, Promotes Flowering in the Orchid Dendrobium Chao Parya Smile. Plant Cell Physiol. 2013, 54, 595-608. doi:10.1093/pcp/pct026.

41. Wang, Y.; Liu, L.; Song, S.; Li, Y.; Shen, L.; Yu, H. DOFT and DOFTIP1 Affect Reproductive Development in the Orchid Dendrobium Chao Praya Smile. J. Exp. Bot. 2017, 68, 5759-5772. doi:10.1093/jxb/erx400.

Publisher's Note: MDPI stays neutral with regard to jurisdictional claims in published maps and institutional affiliations.

(C) 2020 by the authors; licensee MDPI, Basel, Switzerland. This article is an open access article distributed under the terms and conditions of the Creative Commons by Attribution (CC-BY) license (http://creativecommons.org/licenses/by/4.0/). 